\title{
Ruled surfaces corresponding to hyper-dual curves
}

\author{
Selahattin Aslan*1 (D), Murat Bekar ${ }^{2}$ (D), Yusuf Yaylı ${ }^{1}$ (D) \\ ${ }^{1}$ Faculty of Science, Department of Mathematics, Ankara University, Ankara, 06100, Turkey \\ ${ }^{2}$ Faculty of Education, Department of Mathematics Education, Gazi University, Ankara, 06500, Turkey
}

\begin{abstract}
In this paper, we give the definition of the concept of unit hyper-dual sphere. We take a subset of this sphere and show that each curve on this subset represents two ruled surfaces in three dimensional real vector space such that these ruled surfaces have a common base curve and their rulings are perpendicular. Finally, we give some examples to illustrate the applications of our main results.
\end{abstract}

Mathematics Subject Classification (2020). 14J26, 53A25, 70B10, 70E15, 65D17, $70 \mathrm{~B} 15$

Keywords. hyper-dual numbers, unit hyper-dual sphere, hyper-dual curves, ruled surfaces, principal curve

\section{Introduction}

Clifford introduced the algebra of dual numbers $\mathbb{D}$ as an extension of real numbers $\mathbb{R}$ [2]. A dual vector is an ordered triple of dual numbers, and the set of all dual vectors is denoted by $\mathbb{D}^{3}$. Dual vectors were first applied in mechanism by Study [19] and Kotelnikov [11]. There exists a one-to-one correspondence (known as E. Study mapping) between the directed lines in 3-dimensional real vector space $\mathbb{R}^{3}$ and the points of unit dual sphere $\mathbb{S}_{\mathbb{D}}^{2}$ (the set of all unit dual vectors).

The algebra of hyper-dual numbers $\tilde{\mathbb{D}}$ was first defined by Fike to overcome some derivative problems in the complex-step derivative approximation [6,7]. Afterwards, this number system is used in derivative calculations [6-9]. Cohen and Shoham showed that a hyper-dual number consists of two dual numbers [3]. Futhermore, they interpreted hyperdual numbers in the sense of Study [19] and Kotelnikov [11], and they used this number system in the motion of multi-body systems [3-5]. Hyper-dual numbers are suitable for software, analysis and design of airspace systems, and robot manipulators $[4,7]$.

A ruled surface is described as a surface swept out by a straight line moving along a curve [15]. The parametric representation of a ruled surface consists of two curves in $\mathbb{R}^{3}$ similar to a curve on unit dual sphere $\mathbb{S}_{\mathbb{D}}^{2}$. Hence, there exists a one-to-one correspendence between the dual curves on $\mathbb{S}_{\mathbb{D}}^{2}$ and the ruled surfaces in $\mathbb{R}^{3}[20]$. Veldkamp gave the

\footnotetext{
*Corresponding Author.

Email addresses: selahattinnaslan@gmail.com (S. Aslan), murat-bekar@hotmail.com (M. Bekar), yayli@science.ankara.edu.tr (Y. Yaylı)

Received: 28.08.2021; Accepted: 06.09.2021
} 
applications of the dual curves on $\mathbb{S}_{\mathbb{D}}^{2}$ to theoretical space kinematic [20]. Afterwards, these curves have been used in motion of the robot end-effector [14,17], in kinematic formulations of the lines trajectories $[12,13]$ and in kinematic generations of the ruled surfaces [18].

In this paper, we give some basic concepts of hyper-dual numbers. We define unit hyper-dual sphere $\mathbb{S}_{\tilde{\mathbb{D}}}^{2}$. Using E. Study mapping, we show that there exists a one-to-one correspondence between the points of $\mathbb{S}_{\tilde{\mathbb{D}}_{1}}^{2}$ (which is a subset of unit hyper-dual sphere $\left.\mathbb{S}_{\tilde{\mathbb{D}}}^{2}\right)$ and any two intersecting perpendicular directed lines in $\mathbb{R}^{3}$. We give the definition of hyper-dual curves on $\mathbb{S}_{\tilde{\mathbb{D}}}^{2}$. By interpreting these curves in the sense of Veldkamp [20], we show that each hyper-dual curve on $\mathbb{S}_{\tilde{\mathbb{D}}_{1}}^{2}$ represents two ruled surfaces in $\mathbb{R}^{3}$. It is observed that these ruled surfaces intersect along a common base curve and their rulings are perpendicular. It is also observed that each dual curve on unit dual sphere $\mathbb{S}_{\mathbb{D}}^{2}$ represents a ruled surface in $\mathbb{R}^{3}$ while each hyper-dual curve on $\mathbb{S}_{\mathbb{\mathbb { D }}_{1}}^{2}$ represents two ruled surfaces in $\mathbb{R}^{3}$ such that these two ruled surfaces intersect along a common base curve. Examples of ruled surfaces are given to illustrate the applications of our results.

\section{Preliminaries}

In this section, definitions and some algebraic properties of the concepts of dual numbers and hyper-dual numbers will be given to provide a background.

\subsection{Dual numbers}

The set of all dual numbers is defined as

$$
\mathbb{D}=\left\{A=a+\varepsilon a^{*}: a, a^{*} \in \mathbb{R}\right\},
$$

where $\varepsilon$ is the dual unit satisfying

$$
\varepsilon \neq 0, \varepsilon^{2}=0 \text { and } r \varepsilon=\varepsilon r \text { for all } r \in \mathbb{R} \text {. }
$$

The square root of a dual number $A=a+\varepsilon a^{*}$ is defined as

$$
\sqrt{A}=\sqrt{a}+\varepsilon \frac{a^{*}}{2 \sqrt{a}}, \text { for } a>0 .
$$

Taylor series expansion of a dual function $f\left(x+\varepsilon x^{*}\right)$ about a point $x+\varepsilon x^{*}=a+\varepsilon a^{*} \in \mathbb{D}$ can be given as

$$
f\left(a+\varepsilon a^{*}\right)=f(a)+\varepsilon a^{*} f^{\prime}(a),
$$

where the prime represents differentiation with respect to $x$ [20], i.e.

$$
f^{\prime}(x)=\frac{d}{d x} f(x)
$$

The set of dual vectors is defined by

$$
\mathbb{D}^{3}=\left\{\hat{A}=\boldsymbol{a}+\varepsilon \boldsymbol{a}^{*}: \boldsymbol{a}, \boldsymbol{a}^{*} \in \mathbb{R}^{3}\right\}
$$

and each element $\hat{A}$ of $\mathbb{D}^{3}$ is called a dual vector.

The scalar and vector products of any dual vectors $\hat{A}=\boldsymbol{a}+\varepsilon \boldsymbol{a}^{*}$ and $\hat{B}=\boldsymbol{b}+\varepsilon \boldsymbol{b}^{*}$ are defined by

$$
\begin{aligned}
\langle\hat{A}, \hat{B}\rangle_{D} & =\langle\boldsymbol{a}, \boldsymbol{b}\rangle+\varepsilon\left(\left\langle\boldsymbol{a}, \boldsymbol{b}^{*}\right\rangle+\left\langle\boldsymbol{a}^{*}, \boldsymbol{b}\right\rangle\right), \\
\hat{A} \times{ }_{D} \hat{B} & =\boldsymbol{a} \times \boldsymbol{b}+\varepsilon\left(\boldsymbol{a} \times \boldsymbol{b}^{*}+\boldsymbol{a}^{*} \times \boldsymbol{b}\right),
\end{aligned}
$$

where " $\langle$,$\rangle " and " \times$ " denote, respectively, the usual scalar and vector products in 3dimensional real vector space $\mathbb{R}^{3}$. 
The modulus of the dual vector $\hat{A}=\boldsymbol{a}+\varepsilon \boldsymbol{a}^{*}$ is defined to be

$$
|\hat{A}|_{D}=\sqrt{\langle\hat{A}, \hat{A}\rangle_{D}}=|\boldsymbol{a}|+\varepsilon \frac{\left\langle\boldsymbol{a}, \boldsymbol{a}^{*}\right\rangle}{|\boldsymbol{a}|}, \text { for }|\boldsymbol{a}| \neq 0 .
$$

If $|\hat{A}|_{D}=1$ (i.e., $|\boldsymbol{a}|=1$ and $\left\langle\boldsymbol{a}, \boldsymbol{a}^{*}\right\rangle=0$ ), then $\hat{A}=\boldsymbol{a}+\varepsilon \boldsymbol{a}^{*}$ is called a unit dual vector.

Unit dual sphere $\mathbb{S}_{\mathbb{D}}^{2}$, consisting of all unit dual vectors, is defined by

$$
\mathbb{S}_{\mathbb{D}}^{2}=\left\{\hat{A}=\boldsymbol{a}+\varepsilon \boldsymbol{a}^{*}:|\hat{A}|_{D}=1, \hat{A} \in \mathbb{D}^{3}\right\} .
$$

Theorem 2.1. [E. Study Mapping] Each point on unit dual sphere $\mathbb{S}_{\mathbb{D}}^{2}$ represents a directed line in $\mathbb{R}^{3}$. In other words, there is a one-to-one correspondence between the points of unit dual sphere $\mathbb{S}_{\mathbb{D}}^{2}$ and the directed lines in $\mathbb{R}^{3}[19]$.

The scalar product of any unit dual vectors $\hat{A}=\boldsymbol{a}+\varepsilon \boldsymbol{a}^{*}$ and $\hat{B}=\boldsymbol{b}+\varepsilon \boldsymbol{b}^{*}$ is

$$
\langle\hat{A}, \hat{B}\rangle_{D}=\cos \varphi=\cos \theta-\varepsilon \theta^{*} \sin \theta,
$$

where $\varphi=\theta+\varepsilon \theta^{*}$ is a dual angle [19]. If $d_{1}$ and $d_{2}$ are the directed lines in $\mathbb{R}^{3}$ corresponding, respectively, to the unit dual vectors $\hat{A}$ and $\hat{B}$, then $\theta$ is the angle between the real vectors $\boldsymbol{a}$ and $\boldsymbol{b}$, and $\left|\theta^{*}\right|$ is the shortest distance between $d_{1}$ and $d_{2}$, see Fig. 1 .

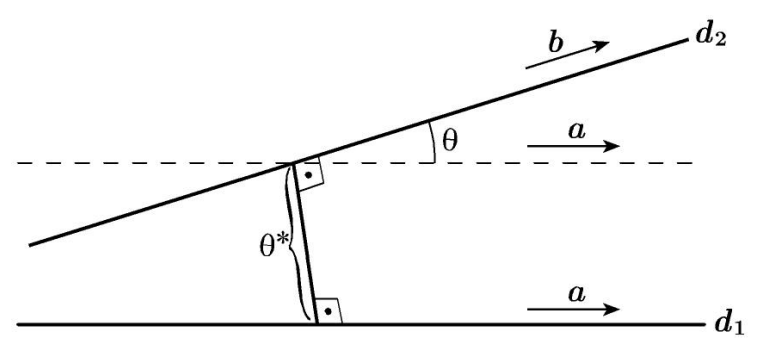

Figure 1. Geometric representation of dual angle $\varphi \in \mathbb{R}^{3}$

The vector product of any unit dual vectors $\hat{A}=\boldsymbol{a}+\varepsilon \boldsymbol{a}^{*}$ and $\hat{B}=\boldsymbol{b}+\varepsilon \boldsymbol{b}^{*}$ is

$$
\hat{A} \times_{D} \hat{B}=\hat{N} \sin \varphi,
$$

where the Taylor series expansion of $\sin \varphi$ is $\sin \varphi=\sin \theta+\varepsilon \theta^{*} \cos \theta$ and where $\hat{N}=$ $\frac{\hat{A} \times_{D} \hat{B}}{\left|\hat{A} \times_{D} \hat{B}\right|_{D}}$ is the common perpendicular direction vector to the dual vectors $\hat{A}$ and $\hat{B}$, directed from $\boldsymbol{a}$ to $\boldsymbol{b}$. For further information about dual numbers, see [1,2,5,20].

\subsection{Hyper-dual numbers}

The set of all hyper-dual numbers is defined as

$$
\tilde{\mathbb{D}}=\left\{\mathbb{A}=a_{0}+\varepsilon_{1} a_{1}+\varepsilon_{2} a_{2}+\varepsilon_{1} \varepsilon_{2} a_{3}: a_{0}, a_{1}, a_{2}, a_{3} \in \mathbb{R}\right\},
$$

where the dual units $\varepsilon_{1}$ and $\varepsilon_{2}$ satisfy

$$
\varepsilon_{1}^{2}=\varepsilon_{2}^{2}=\left(\varepsilon_{1} \varepsilon_{2}\right)^{2}=0 \text { and } \varepsilon_{1} \neq \varepsilon_{2}, \varepsilon_{1} \neq 0, \varepsilon_{2} \neq 0, \varepsilon_{1} \varepsilon_{2}=\varepsilon_{2} \varepsilon_{1} \neq 0 .
$$

The algebra of $\tilde{\mathbb{D}}$ can be embedded in the real exterior algebra $\wedge V$ where $V$ is a real vector space with an orthogonal basis $e_{1}, e_{2}, e_{3}, e_{4}$, as follows: let $\varepsilon_{1}=e_{1} \wedge e_{2}$ and $\varepsilon_{2}=e_{3} \wedge e_{4}$. Then, one can recover the algebra of the $\tilde{\mathbb{D}}$ as this 4-dimensional subalgebra of the exterior algebra $\wedge V$ that is spanned by $\left\{1, \varepsilon_{1}, \varepsilon_{2}, \varepsilon_{1} \varepsilon_{2}\right\}$.

Addition and multiplication of any hyper-dual numbers $\mathbb{A}=a_{0}+\varepsilon_{1} a_{1}+\varepsilon_{2} a_{2}+\varepsilon_{1} \varepsilon_{2} a_{3}$ and $\mathbb{B}=b_{0}+\varepsilon_{1} b_{1}+\varepsilon_{2} b_{2}+\varepsilon_{1} \varepsilon_{2} b_{3}$ are defined, respectively, as

$$
\mathbb{A}+\mathbb{B}=\left(a_{0}+b_{0}\right)+\varepsilon_{1}\left(a_{1}+b_{1}\right)+\varepsilon_{2}\left(a_{2}+b_{2}\right)+\varepsilon_{1} \varepsilon_{2}\left(a_{3}+b_{3}\right),
$$




$$
\begin{aligned}
\mathbb{A B} & =\left(a_{0} b_{0}\right)+\varepsilon_{1}\left(a_{0} b_{1}+a_{1} b_{0}\right)+\varepsilon_{2}\left(a_{0} b_{2}+a_{2} b_{0}\right) \\
& +\varepsilon_{1} \varepsilon_{2}\left(a_{0} b_{3}+a_{1} b_{2}+a_{2} b_{1}+a_{3} b_{0}\right) .
\end{aligned}
$$

The multiplicative-inverse of a hyper-dual number $\mathbb{A}=a_{0}+\varepsilon_{1} a_{1}+\varepsilon_{2} a_{2}+\varepsilon_{1} \varepsilon_{2} a_{3}$ is

$$
\mathbb{A}^{-1}=\frac{1}{\mathbb{A}}=\frac{1}{a_{0}}-\varepsilon_{1} \frac{a_{1}}{a_{0}^{2}}-\varepsilon_{2} \frac{a_{2}}{a_{0}^{2}}+\varepsilon_{1} \varepsilon_{2}\left(-\frac{a_{3}}{a_{0}^{2}}+\frac{2 a_{1} a_{2}}{a_{0}^{3}}\right), \quad \text { if } a_{0} \neq 0 .
$$

Thus, a hyper-dual number in the form $\mathbb{A}=0+\varepsilon_{1} a_{1}+\varepsilon_{2} a_{2}+\varepsilon_{1} \varepsilon_{2} a_{3}=\varepsilon_{1} a_{1}+\varepsilon_{2} a_{2}+\varepsilon_{1} \varepsilon_{2} a_{3}$ does not have an inverse.

Taylor series expansion of a hyper-dual function $f\left(x_{0}+\varepsilon_{1} x_{1}+\varepsilon_{2} x_{2}+\varepsilon_{1} \varepsilon_{2} x_{3}\right)$ about a point $x_{0}+\varepsilon_{1} x_{1}+\varepsilon_{2} x_{2}+\varepsilon_{1} \varepsilon_{2} x_{3}=a_{0}+\varepsilon_{1} a_{1}+\varepsilon_{2} a_{2}+\varepsilon_{1} \varepsilon_{2} a_{3} \in \tilde{\mathbb{D}}$ can be given as

$$
\begin{aligned}
f\left(a_{0}+\varepsilon_{1} a_{1}+\varepsilon_{2} a_{2}+\varepsilon_{1} \varepsilon_{2} a_{3}\right) & =f\left(a_{0}\right)+\varepsilon_{1} a_{1} f^{\prime}\left(a_{0}\right)+\varepsilon_{2} a_{2} f^{\prime}\left(a_{0}\right) \\
& +\varepsilon_{1} \varepsilon_{2}\left(a_{3} f^{\prime}\left(a_{0}\right)+a_{1} a_{2} f^{\prime \prime}\left(a_{0}\right)\right),
\end{aligned}
$$

where the prime represents differentiation with respect to $x_{0}$, i.e.

$$
f^{\prime}\left(x_{0}\right)=\frac{d}{d x_{0}} f\left(x_{0}\right),
$$

see $[6-9]$.

A hyper-dual number $\mathbb{A}=a_{0}+\varepsilon_{1} a_{1}+\varepsilon_{2} a_{2}+\varepsilon_{1} \varepsilon_{2} a_{3}$ can be given in terms of two dual numbers as

$$
\mathbb{A}=A+\varepsilon^{*} A^{*},
$$

where $\varepsilon_{1}=\varepsilon, \varepsilon_{2}=\varepsilon^{*}$ and $A=a_{0}+\varepsilon a_{1}, A^{*}=a_{2}+\varepsilon a_{3} \in \mathbb{D}$.

The addition and multiplication rules of two hyper-dual numbers $\mathbb{A}=a_{0}+\varepsilon_{1} a_{1}+\varepsilon_{2} a_{2}+$ $\varepsilon_{1} \varepsilon_{2} a_{3}=A+\varepsilon^{*} A^{*}$ and $\mathbb{B}=b_{0}+\varepsilon_{1} b_{1}+\varepsilon_{2} b_{2}+\varepsilon_{1} \varepsilon_{2} b_{3}=B+\varepsilon^{*} B^{*}$ given, respectively, by Eqs. (2.15) and (2.16) can be expressed differently as

$$
\begin{aligned}
\mathbb{A}+\mathbb{B} & =(A+B)+\varepsilon^{*}\left(A^{*}+B^{*}\right), \\
\mathbb{A} \mathbb{B} & =A B+\varepsilon^{*}\left(A B^{*}+A^{*} B\right) .
\end{aligned}
$$

An alternative representation of the multiplicative-inverse of a hyper-dual number $\mathbb{A}=$ $a_{0}+\varepsilon_{1} a_{1}+\varepsilon_{2} a_{2}+\varepsilon_{1} \varepsilon_{2} a_{3}=A+\varepsilon^{*} A^{*}$ given by Eq. (2.17) can be given as

$$
\mathbb{A}^{-1}=\frac{1}{A}-\varepsilon^{*} \frac{A^{*}}{A^{2}}, \text { for } a_{0} \neq 0 .
$$

This means that a hyper-dual number $\mathbb{A}=A+\varepsilon^{*} A^{*}$ providing $A=0+\varepsilon a_{1}=\varepsilon a_{1}$ does not have an inverse.

If we extend the real vectors $\boldsymbol{a}$ and $\boldsymbol{p} \times \boldsymbol{a}$ in a dual vector $\hat{A}=\boldsymbol{a}+\varepsilon(\boldsymbol{p} \times \boldsymbol{a})$, respectively, to the dual vectors $\hat{A}$ and $\hat{P} \times_{D} \hat{A}$ then we obtain the hyper-dual vector

$$
\widetilde{\mathbb{A}}=\hat{A}+\varepsilon^{*}\left(\hat{P} \times_{D} \hat{A}\right) .
$$

Scalar and vector products of any hyper-dual vectors $\widetilde{\mathbb{A}}=\hat{A}+\varepsilon^{*}\left(\hat{P} \times{ }_{D} \hat{A}\right)$ and $\widetilde{\mathbb{B}}=$ $\hat{B}+\varepsilon^{*}\left(\hat{K} \times{ }_{D} \hat{B}\right)$ can be given, respectively, as

$$
\begin{aligned}
\langle\widetilde{\mathbb{A}}, \widetilde{\mathbb{B}}\rangle_{H D} & =|\hat{A}|_{D}|\hat{B}|_{D} \cos \tilde{\varphi} \\
\widetilde{\mathbb{A}} \times_{H D} \widetilde{\mathbb{B}} & =|\hat{A}|_{D}|\hat{B}|_{D} \boldsymbol{n} \sin \tilde{\varphi},
\end{aligned}
$$

where $\tilde{\varphi}$ is a hyper-dual angle and $\boldsymbol{n}$ is the common perpendicular direction vector to the hyper-dual vectors $\widetilde{\mathbb{A}}$ and $\widetilde{\mathbb{B}}$, directed from $\hat{A}$ to $\hat{B}$. For further information about hyper-dual numbers, see [3-5]. 


\section{Hyper-dual numbers and ruled surfaces}

In this section, we express some basic concepts of hyper-dual numbers. Using these expressions, we define a subset $\mathbb{S}_{\tilde{\mathbb{D}}_{1}}^{2}$ of unit hyper-dual sphere $\mathbb{S}_{\tilde{\mathbb{D}}}^{2}$ such that each element of $\mathbb{S}_{\tilde{\mathbb{D}}_{1}}^{2}$ represents two intersecting and perpendicular directed lines in $\mathbb{R}^{3}$. Moreover, we show that each hyper-dual curve on $\mathbb{S}_{\mathbb{\mathbb { D }}_{1}}^{2}$ represents two ruled surfaces in $\mathbb{R}^{3}$. These ruled surfaces have a common base curve and their rulings are perpendicular.

\subsection{Some basic concepts of hyper-dual numbers}

The square root of a hyper-dual number $\mathbb{A}=A+\varepsilon^{*} A^{*}$ can be defined by

or

$$
\sqrt{\mathbb{A}}=\sqrt{A}+\varepsilon^{*} \frac{A^{*}}{2 \sqrt{A}}, \text { for } a_{0}>0
$$

$$
\sqrt{\mathbb{A}}=\sqrt{a_{0}}+\varepsilon \frac{a_{1}}{2 \sqrt{a_{0}}}+\varepsilon^{*} \frac{a_{2}}{2 \sqrt{a_{0}}}+\varepsilon \varepsilon^{*}\left(\frac{a_{3}}{2 \sqrt{a_{0}}}-\frac{a_{1} a_{2}}{4 a_{0} \sqrt{a_{0}}}\right) \text {, for } a_{0}>0 .
$$

The set of all hyper-dual vectors is defined to be

$$
\begin{aligned}
\tilde{\mathbb{D}}^{3} & =\left\{\widetilde{\mathbb{A}}=\hat{A}+\varepsilon^{*} \hat{A}^{*}: \hat{A}, \hat{A}^{*} \in \mathbb{D}^{3}\right\} \\
& =\left\{\widetilde{\mathbb{A}}=\boldsymbol{a}_{0}+\varepsilon \boldsymbol{a}_{1}+\varepsilon^{*} \boldsymbol{a}_{2}+\varepsilon \varepsilon^{*} \boldsymbol{a}_{3}: \boldsymbol{a}_{0}, \boldsymbol{a}_{1}, \boldsymbol{a}_{2}, \boldsymbol{a}_{3} \in \mathbb{R}^{3}\right\},
\end{aligned}
$$

and each element $\widetilde{\mathbb{A}}$ of $\tilde{\mathbb{D}}^{3}$ is called a hyper-dual vector.

The scalar and vector products of any hyper-dual vectors $\widetilde{\mathbb{A}}=\hat{A}+\varepsilon^{*} \hat{A}^{*}=\boldsymbol{a}_{0}+\varepsilon \boldsymbol{a}_{1}+$ $\varepsilon^{*} \boldsymbol{a}_{2}+\varepsilon \varepsilon^{*} \boldsymbol{a}_{3}$ and $\widetilde{B}=\hat{B}+\varepsilon^{*} \hat{B}^{*}=\boldsymbol{b}_{0}+\varepsilon \boldsymbol{b}_{1}+\varepsilon^{*} \boldsymbol{b}_{2}+\varepsilon \varepsilon^{*} \boldsymbol{b}_{3}$ are defined, respectively, by

$$
\begin{aligned}
\langle\widetilde{\mathbb{A}}, \widetilde{\mathbb{B}}\rangle_{H D} & =\langle\hat{A}, \hat{B}\rangle_{D}+\varepsilon^{*}\left(\left\langle\hat{A}, \hat{B}^{*}\right\rangle_{D}+\left\langle\hat{A}^{*}, \hat{B}\right\rangle_{D}\right) \\
& =\left\langle\boldsymbol{a}_{0}, \boldsymbol{b}_{0}\right\rangle+\varepsilon\left(\left\langle\boldsymbol{a}_{0}, \boldsymbol{b}_{1}\right\rangle+\left\langle\boldsymbol{a}_{1}, \boldsymbol{b}_{0}\right\rangle\right)+\varepsilon^{*}\left(\left\langle\boldsymbol{a}_{0}, \boldsymbol{b}_{2}\right\rangle+\left\langle\boldsymbol{a}_{2}, \boldsymbol{b}_{0}\right\rangle\right) \\
& +\varepsilon \varepsilon^{*}\left(\left\langle\boldsymbol{a}_{0}, \boldsymbol{b}_{3}\right\rangle+\left\langle\boldsymbol{a}_{1}, \boldsymbol{b}_{2}\right\rangle+\left\langle\boldsymbol{a}_{2}, \boldsymbol{b}_{1}\right\rangle+\left\langle\boldsymbol{a}_{3}, \boldsymbol{b}_{0}\right\rangle\right), \\
\widetilde{\mathbb{A}} \times_{H D} \widetilde{\mathbb{B}} & =\hat{A} \times_{D} \hat{B}+\varepsilon^{*}\left(\hat{A} \times_{D} \hat{B}^{*}+\hat{A}^{*} \times{ }_{D} \hat{B}\right) \\
& =\boldsymbol{a}_{0} \times \boldsymbol{b}_{0}+\varepsilon\left(\boldsymbol{a}_{0} \times \boldsymbol{b}_{1}+\boldsymbol{a}_{1} \times \boldsymbol{b}_{0}\right)+\varepsilon^{*}\left(\boldsymbol{a}_{0} \times \boldsymbol{b}_{2}+\boldsymbol{a}_{2} \times \boldsymbol{b}_{0}\right) \\
& +\varepsilon \varepsilon^{*}\left(\boldsymbol{a}_{0} \times \boldsymbol{b}_{3}+\boldsymbol{a}_{1} \times \boldsymbol{b}_{2}+\boldsymbol{a}_{2} \times \boldsymbol{b}_{1}+\boldsymbol{a}_{3} \times \boldsymbol{b}_{0}\right) .
\end{aligned}
$$

It is obvious that $\langle\widetilde{\mathbb{A}}, \widetilde{\mathbb{B}}\rangle_{H D}$ and $\widetilde{\mathbb{A}} \times_{H D} \widetilde{\mathbb{B}}$ are, respectively, a hyper-dual number and a hyper-dual vector.

The norm of a hyper-dual vector $\widetilde{\mathbb{A}}=\hat{A}+\varepsilon^{*} \hat{A}^{*}=\boldsymbol{a}_{0}+\varepsilon \boldsymbol{a}_{1}+\varepsilon^{*} \boldsymbol{a}_{2}+\varepsilon \varepsilon^{*} \boldsymbol{a}_{3}$ is defined to be

$$
\begin{aligned}
N_{\widetilde{\mathbb{A}}} & =\langle\widetilde{\mathbb{A}}, \widetilde{\mathbb{A}}\rangle_{H D}=|\hat{A}|_{D}^{2}+2 \varepsilon^{*}\left\langle\hat{A}, \hat{A}^{*}\right\rangle_{D} \\
& =\left|\boldsymbol{a}_{0}\right|^{2}+2\left(\varepsilon\left\langle\boldsymbol{a}_{0}, \boldsymbol{a}_{1}\right\rangle+\varepsilon^{*}\left\langle\boldsymbol{a}_{0}, \boldsymbol{a}_{2}\right\rangle+\varepsilon \varepsilon^{*}\left(\left\langle\boldsymbol{a}_{0}, \boldsymbol{a}_{3}\right\rangle+\left\langle\boldsymbol{a}_{1}, \boldsymbol{a}_{2}\right\rangle\right)\right) .
\end{aligned}
$$

The modulus (i.e., square root of the norm) of the hyper-dual vector $\widetilde{\mathbb{A}}$ is also defined to be

$$
\begin{aligned}
|\widetilde{\mathbb{A}}|_{H D} & =\sqrt{\langle\widetilde{\mathbb{A}}, \widetilde{\mathbb{A}}\rangle_{H D}}=|\hat{A}|_{D}+\varepsilon^{*} \frac{\left\langle\hat{A}, \hat{A}^{*}\right\rangle_{D}}{|\hat{A}|_{D}} \\
& =\left|\boldsymbol{a}_{0}\right|+\varepsilon \frac{\left\langle\boldsymbol{a}_{0}, \boldsymbol{a}_{1}\right\rangle}{\left|\boldsymbol{a}_{0}\right|}+\varepsilon^{*} \frac{\left\langle\boldsymbol{a}_{0}, \boldsymbol{a}_{2}\right\rangle}{\left|\boldsymbol{a}_{0}\right|} \\
& +\varepsilon \varepsilon^{*}\left(\frac{\left\langle\boldsymbol{a}_{0}, \boldsymbol{a}_{3}\right\rangle}{\left|\boldsymbol{a}_{0}\right|}+\frac{\left\langle\boldsymbol{a}_{1}, \boldsymbol{a}_{2}\right\rangle}{\left|\boldsymbol{a}_{0}\right|}-\frac{\left\langle\boldsymbol{a}_{0}, \boldsymbol{a}_{1}\right\rangle\left\langle\boldsymbol{a}_{0}, \boldsymbol{a}_{2}\right\rangle}{\left|\boldsymbol{a}_{0}\right|^{3}}\right),
\end{aligned}
$$


where $\left|\boldsymbol{a}_{0}\right| \neq 0$.

If $|\widetilde{\mathbb{A}}|_{H D}=1$ (i.e., $|\hat{A}|_{D}=1$ and $\left\langle\hat{A}, \hat{A}^{*}\right\rangle_{D}=0$ ), then $\widetilde{\mathbb{A}}=\hat{A}+\varepsilon^{*} \hat{A}^{*}$ is called a unit hyper-dual vector.

Definition 3.1. [Unit hyper-dual sphere] Unit hyper-dual sphere $\mathbb{S}_{\tilde{\mathbb{D}}}^{2}$, consisting of all unit hyper-dual vectors, is defined as

$$
\mathbb{S}_{\tilde{\mathbb{D}}}^{2}=\left\{\widetilde{\mathbb{A}}=\hat{A}+\varepsilon^{*} \hat{A}^{*}:|\widetilde{\mathbb{A}}|_{H D}=1 ; \quad \hat{A}, \hat{A}^{*} \in \mathbb{D}^{3}\right\} .
$$

Theorem 3.2. Let us take a subset of unit hyper-dual sphere $\mathbb{S}_{\tilde{\mathbb{D}}}^{2}$ as

$$
\mathbb{S}_{\widetilde{\mathbb{D}}_{1}}^{2}=\left\{\widetilde{\mathbb{A}}=\hat{A}+\varepsilon^{*} \hat{A}^{*}:\left|\hat{A}^{*}\right|_{D}=1, \widetilde{\mathbb{A}} \in \mathbb{S}_{\widetilde{\mathbb{D}}}^{2}\right\} \subset \mathbb{S}_{\widetilde{\mathbb{D}}}^{2} .
$$

Then, there exists a one-to-one correspondence between the points of $\mathbb{S}_{\tilde{\mathbb{D}}_{1}}^{2}$ and any two intersecting perpendicular directed lines in $\mathbb{R}^{3}$.

Proof. Since $\widetilde{\mathbb{A}} \in \mathbb{S}_{\widetilde{\mathbb{D}}_{1}}^{2}, \hat{A}$ and $\hat{A}^{*}$ are unit dual vectors and $\widetilde{\mathbb{A}}=\hat{A}+\varepsilon^{*} \hat{A}^{*}$ is a unit hyperdual vector satisfying $|\hat{A}|_{D}=1$ and $\left\langle\hat{A}, \hat{A}^{*}\right\rangle_{D}=0$. According to Theorem 2.1 , the unit dual vectors $\hat{A}$ and $\hat{A}^{*}$ represent the directed lines $d_{1}$ and $d_{2}$ in $\mathbb{R}^{3}$, respectively. Using Eq. (2.11), the dual angle $\varphi=\theta+\varepsilon \theta^{*}$ between $\hat{A}$ and $\hat{A}^{*}$ can be given as

$$
\left\langle\hat{A}, \hat{A}^{*}\right\rangle_{D}=\cos \theta-\varepsilon \theta^{*} \sin \theta=\cos \varphi .
$$

From $\left\langle\hat{A}, \hat{A}^{*}\right\rangle_{D}=0$, we get $\theta=\frac{\pi}{2}$ and $\theta^{*}=0$. Thus, the lines $d_{1}$ and $d_{2}$ are perpendicular and intersecting in $\mathbb{R}^{3}$.

\subsection{Ruled surfaces constructed by hyper-dual curves on $\mathbb{S}_{\tilde{\mathbb{D}}_{1}}^{2}$}

A ruled surface in $\mathbb{R}^{3}$ is a surface swept out by a straight line moving along a curve. The various positions of the generating line are called the rulings of the surface. Such a surface can be given by the parametrization

$$
\Phi(t, v)=\beta(t)+v \gamma(t), \quad t \in I=(a, b) \subset \mathbb{R}, \quad v \in \mathbb{R} .
$$

Here; $\beta(t)$ is the base curve of $\Phi(t, v)$ and the unit vector $\gamma(t)$ is the director curve of $\Phi(t, v)[15]$.

A dual curve in $\mathbb{D}^{3}$ can be defined as

$$
\begin{aligned}
\hat{\Gamma}: I \subset \mathbb{R} \longrightarrow \mathbb{D}^{3} & \\
t \longrightarrow \hat{\Gamma}(t) & =\left(a_{1}(t)+\varepsilon a_{1}^{*}(t), a_{2}(t)+\varepsilon a_{2}^{*}(t), a_{3}(t)+\varepsilon a_{3}^{*}(t)\right) \\
& =\boldsymbol{a}(t)+\varepsilon \boldsymbol{a}^{*}(t),
\end{aligned}
$$

where $I$ is an open interval in $\mathbb{R}$ and $\boldsymbol{a}(t)=\left(a_{1}(t), a_{2}(t), a_{3}(t)\right), \boldsymbol{a}^{*}(t)=\left(a_{1}^{*}(t), a_{2}^{*}(t), a_{3}^{*}(t)\right) \in$ $\mathbb{R}^{3}$. If every real valued functions $a_{i}(t)$ and $a_{i}^{*}(t)$ are differentiable for $i=1,2,3$, then the dual space curve $\hat{\Gamma}(t)$ is differentiable. And if $|\hat{\Gamma}(t)|_{D}=1$, then the dual curve $\hat{\Gamma}(t)$ is on unit dual sphere $\mathbb{S}_{\mathbb{D}}^{2}[16]$.

Let $\hat{\Gamma}(t)=\boldsymbol{a}(t)+\varepsilon \boldsymbol{a}^{*}(t)$ be a dual curve on the unit dual sphere $\mathbb{S}_{\mathbb{D}}^{2}$. Then, the ruled surface corresponding to the dual curve $\hat{\Gamma}(t)$ can be given in $\mathbb{R}^{3}$ as

$$
\Phi(t, u)=\boldsymbol{a}(t) \times \boldsymbol{a}^{*}(t)+u \boldsymbol{a}(t), \quad t \in I \subset \mathbb{R}, \quad u \in \mathbb{R}
$$

where $\alpha(t)=\boldsymbol{a}(t) \times \boldsymbol{a}^{*}(t)$ is the base curve and $\boldsymbol{a}(t)$ is the director curve of $\Phi(t, u)[10,20]$.

Definition 3.3. [Hyper-dual curve] A hyper-dual curve in $\tilde{\mathbb{D}}^{3}$ can be defined as

$$
\begin{aligned}
\tilde{\Gamma}: I \subset \mathbb{R} & \longrightarrow \tilde{\mathbb{D}}^{3} \\
t & \longrightarrow \tilde{\Gamma}(t)=\hat{A}(t)+\varepsilon^{*} \hat{A}^{*}(t)
\end{aligned}
$$


where $I$ is an open interval in $\mathbb{R}$. If $\hat{A}(t)$ and $\hat{A}^{*}(t)$ are differentiable dual curves in $\mathbb{D}^{3}$, then the hyper-dual curve $\tilde{\Gamma}(t)$ in $\tilde{\mathbb{D}}^{3}$ is differentiable. And if $|\tilde{\Gamma}(t)|_{H D}=1$, then $\tilde{\Gamma}(t)$ is a hyper-dual curve on unit hyper-dual sphere $\mathbb{S}_{\tilde{\mathbb{D}}}^{2}$. Moreover if $\tilde{\Gamma}(t)$ is a hyper-dual curve on $\mathbb{S}_{\tilde{\mathbb{D}}}^{2}$ and $\left|\hat{A}^{*}(t)\right|_{D}=1$, then $\tilde{\Gamma}(t)$ is a hyper-dual curve on $\mathbb{S}_{\tilde{\mathbb{D}}_{1}}^{2}$.

Theorem 3.4. Let $\tilde{\Gamma}(t)=\hat{A}(t)+\varepsilon^{*} \hat{A}^{*}(t)$ be a hyper-dual curve on $\mathbb{S}_{\tilde{\mathbb{D}}_{1}}^{2}$. Then, each hyper-dual curve $\tilde{\Gamma}(t)$ represents two ruled surfaces in $\mathbb{R}^{3}$ such that these surfaces have a common base curve and the position vectors of their director curves are perpendicular.

Proof. Since $\tilde{\Gamma}(t)=\hat{A}(t)+\varepsilon^{*} \hat{A}^{*}(t)$ is a hyper-dual curve on $\mathbb{S}_{\tilde{\mathbb{D}}_{1}}^{2}, \hat{A}(t)$ and $\hat{A}^{*}(t)$ are dual curves on unit dual sphere $\mathbb{S}_{\mathbb{D}}^{2}$. These curves $\hat{A}(t)$ and $\hat{A}^{*}(t)$ can be expressed as

$$
\hat{A}(t)=\boldsymbol{a}_{0}(t)+\varepsilon \boldsymbol{a}_{1}(t) \text { and } \hat{A}^{*}(t)=\boldsymbol{a}_{2}(t)+\varepsilon \boldsymbol{a}_{3}(t),
$$

where $\boldsymbol{a}_{0}(t), \boldsymbol{a}_{1}(t), \boldsymbol{a}_{2}(t), \boldsymbol{a}_{3}(t) \in \mathbb{R}^{3}$. The scalar product of $\hat{A}(t)=\boldsymbol{a}_{0}(t)+\varepsilon \boldsymbol{a}_{1}(t)$ and $\hat{A}^{*}(t)=\boldsymbol{a}_{2}(t)+\varepsilon \boldsymbol{a}_{3}(t)$ is

$$
\left\langle\hat{A}(t), \hat{A}^{*}(t)\right\rangle_{D}=\left\langle\boldsymbol{a}_{0}(t), \boldsymbol{a}_{2}(t)\right\rangle+\varepsilon\left(\left\langle\boldsymbol{a}_{0}(t), \boldsymbol{a}_{3}(t)\right\rangle+\left\langle\boldsymbol{a}_{1}(t), \boldsymbol{a}_{2}(t)\right\rangle\right) .
$$

Since $\tilde{\Gamma}(t)$ is a hyper-dual curve on $\mathbb{S}_{\tilde{\mathbb{D}}_{1}}^{2}$, it is also a hyper-dual curve on $\mathbb{S}_{\tilde{\mathbb{D}}}^{2}$, and thus $\left\langle\hat{A}(t), \hat{A}^{*}(t)\right\rangle_{D}=0$. This means that

$$
\left\langle\boldsymbol{a}_{0}(t), \boldsymbol{a}_{2}(t)\right\rangle=0 \text { and }\left\langle\boldsymbol{a}_{0}(t), \boldsymbol{a}_{3}(t)\right\rangle+\left\langle\boldsymbol{a}_{1}(t), \boldsymbol{a}_{2}(t)\right\rangle=0 .
$$

Using Eq. (3.18), the ruled surfaces corresponding to $\hat{A}(t)=\boldsymbol{a}_{0}(t)+\varepsilon \boldsymbol{a}_{1}(t)$ and $\hat{A}^{*}(t)=$ $\boldsymbol{a}_{2}(t)+\varepsilon \boldsymbol{a}_{3}(t)$ can be given, respectively, as

$$
\begin{array}{ll}
\Phi_{1}\left(t, u_{1}\right)=\boldsymbol{a}_{0}(t) \times \boldsymbol{a}_{1}(t)+u_{1} \boldsymbol{a}_{0}(t), & u_{1} \in \mathbb{R}, \\
\Phi_{2}\left(t, u_{2}\right)=\boldsymbol{a}_{2}(t) \times \boldsymbol{a}_{3}(t)+u_{2} \boldsymbol{a}_{2}(t), & u_{2} \in \mathbb{R},
\end{array}
$$

where $\alpha_{1}(t)=\boldsymbol{a}_{0}(t) \times \boldsymbol{a}_{1}(t)$ and $\alpha_{2}(t)=\boldsymbol{a}_{2}(t) \times \boldsymbol{a}_{3}(t)$ are the base curves of $\Phi_{1}\left(t, u_{1}\right)$ and $\Phi_{2}\left(t, u_{2}\right)$, respectively. Also, $\boldsymbol{a}_{0}(t)$ and $\boldsymbol{a}_{2}(t)$ are the director curves of $\Phi_{1}\left(t, u_{1}\right)$ and $\Phi_{2}\left(t, u_{2}\right)$, recpectively.

For $t=t_{0}$, let us denote $\Phi_{1}\left(t_{0}, u_{1}\right)$ by the line $m_{t_{0}}\left(u_{1}\right)$ and $\Phi_{2}\left(t_{0}, u_{2}\right)$ by the line $n_{t_{0}}\left(u_{2}\right)$. It is obvious that $m_{t}\left(u_{1}\right)$ and $n_{t}\left(u_{2}\right)$ are, recpectively, the rulings of the surfaces $\Phi_{1}\left(t, u_{1}\right)$ and $\Phi_{2}\left(t, u_{2}\right)$, for all $t \in I$. Moreover, $m_{t_{0}}\left(u_{1}\right)$ is a line corresponding to the unit dual vector $\hat{A}\left(t_{0}\right)=\boldsymbol{a}_{0}\left(t_{0}\right)+\varepsilon \boldsymbol{a}_{1}\left(t_{0}\right)$ and $n_{t_{0}}\left(u_{2}\right)$ is a line corresponding to the unit dual vector $\hat{A}^{*}\left(t_{0}\right)=\boldsymbol{a}_{2}\left(t_{0}\right)+\varepsilon \boldsymbol{a}_{3}\left(t_{0}\right)$, where $\boldsymbol{a}_{0}\left(t_{0}\right)$ and $\boldsymbol{a}_{2}\left(t_{0}\right)$ are the direction vectors of $m_{t_{0}}\left(u_{1}\right)$ and $n_{t_{0}}\left(u_{2}\right)$, respectively.

Since $\tilde{\Gamma}\left(t_{0}\right)=\hat{A}\left(t_{0}\right)+\varepsilon^{*} \hat{A}^{*}\left(t_{0}\right) \in \mathbb{S}_{\tilde{\mathbb{D}}_{1}}^{2}, \tilde{\Gamma}\left(t_{0}\right)$ represents two intersecting perpendicular lines (which are $m_{t_{0}}\left(u_{1}\right)$ and $n_{t_{0}}\left(u_{2}\right)$ ) in $\mathbb{R}^{3}$. Let us denote the intersection point of the lines $m_{t}\left(u_{1}\right)$ and $n_{t}\left(u_{2}\right)$ by $k(t)$, for all $t \in I$. Then, according to E. Study mapping the moments of the vectors $\boldsymbol{a}_{0}(t)$ and $\boldsymbol{a}_{2}(t)$ with respect to the origin $O$ can be given as

$$
\begin{aligned}
& \boldsymbol{a}_{1}(t)=k(t) \times \boldsymbol{a}_{0}(t), \\
& \boldsymbol{a}_{3}(t)=k(t) \times \boldsymbol{a}_{2}(t),
\end{aligned}
$$

respectively. Inserting Eq. (3.25) in Eq. (3.23), we get

$$
\begin{aligned}
\Phi_{1}\left(t, u_{1}\right) & =\boldsymbol{a}_{0}(t) \times \boldsymbol{a}_{1}(t)+u_{1} \boldsymbol{a}_{0}(t) \\
& =\boldsymbol{a}_{0}(t) \times\left(k(t) \times \boldsymbol{a}_{0}(t)\right)+u_{1} \boldsymbol{a}_{0}(t) \\
& =\left\langle\boldsymbol{a}_{0}(t), \boldsymbol{a}_{0}(t)\right\rangle k(t)-\left\langle\boldsymbol{a}_{0}(t), k(t)\right\rangle \boldsymbol{a}_{0}(t)+u_{1} \boldsymbol{a}_{0}(t) \\
& =k(t)-\left\langle\boldsymbol{a}_{0}(t), k(t)\right\rangle \boldsymbol{a}_{0}(t)+u_{1} \boldsymbol{a}_{0}(t) \\
& =k(t)+\left(u_{1}-\left\langle\boldsymbol{a}_{0}(t), k(t)\right\rangle\right) \boldsymbol{a}_{0}(t),
\end{aligned}
$$


where $\left\langle\boldsymbol{a}_{0}(t), \boldsymbol{a}_{0}(t)\right\rangle=1$. And inserting $v_{1}=u_{1}-\left\langle\boldsymbol{a}_{0}(t), k(t)\right\rangle$ in Eq. (3.27), we also get Eq. (3.23) as

$$
\Phi_{1}\left(t, v_{1}\right)=k(t)+v_{1} \boldsymbol{a}_{0}(t), \quad v_{1} \in \mathbb{R} .
$$

Similarly, we can obtain Eq. (3.24) as

$$
\Phi_{2}\left(t, v_{2}\right)=k(t)+v_{2} \boldsymbol{a}_{2}(t), \quad v_{2} \in \mathbb{R} .
$$

From Eqs. (3.28) and (3.29), it can be seen that ruled surfaces $\Phi_{1}\left(t, v_{1}\right)$ and $\Phi_{2}\left(t, v_{2}\right)$ possess a common base curve that is $k(t)$. And from Eq. (3.22), it can be seen that the position vectors of the director curves $\boldsymbol{a}_{0}(t)$ and $\boldsymbol{a}_{2}(t)$ of the surfaces $\Phi_{1}\left(t, v_{1}\right)$ and $\Phi_{2}\left(t, v_{2}\right)$ are perpendicular, see Fig. 2.

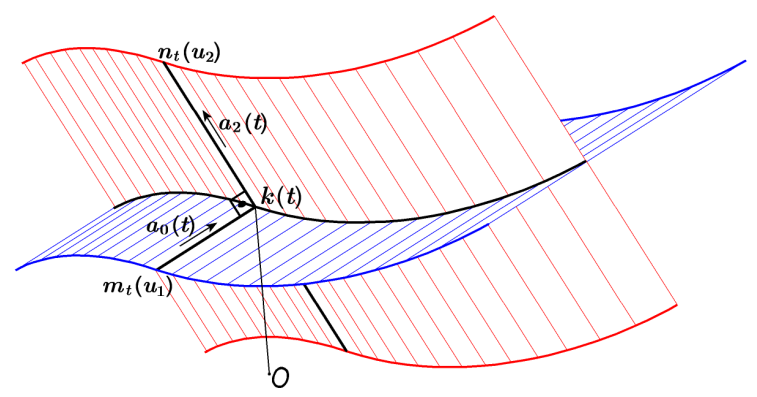

Figure 2. Geometric representation of two ruled surfaces in $\mathbb{R}^{3}$ corresponding to the hyper-dual curve $\tilde{\Gamma}(t)$ on $\mathbb{S}_{\tilde{\mathbb{D}}_{1}}^{2}$.

Theorem 3.5. Let $\Phi_{1}\left(t, v_{1}\right)=k(t)+v_{1} \boldsymbol{a}_{0}(t)$ and $\Phi_{2}\left(t, v_{2}\right)=k(t)+v_{2} \boldsymbol{a}_{2}(t)$ be the ruled surfaces corresponding to the hyper-dual curve $\tilde{\Gamma}(t)=\hat{A}(t)+\varepsilon^{*} \hat{A}^{*}(t)$ on $\mathbb{S}_{\tilde{\mathbb{D}}_{1}}^{2}$, where $\hat{A}(t)=$ $\boldsymbol{a}_{0}(t)+\varepsilon \boldsymbol{a}_{1}(t)$ and $\hat{A}^{*}(t)=\boldsymbol{a}_{2}(t)+\varepsilon \boldsymbol{a}_{3}(t)$. Then, the normal vectors of the surfaces $\Phi_{1}\left(t, v_{1}\right)$ and $\Phi_{2}\left(t, v_{2}\right)$ are perpendicular along the common base curve $k(t)$ if and only if the velocity vector $\frac{d}{d t} k(t)=k^{\prime}(t)$ is perpendicular to $\boldsymbol{a}_{0}(t)$ or $\boldsymbol{a}_{2}(t)$.

Proof. The normal vectors of $\Phi_{1}\left(t, v_{1}\right)$ and $\Phi_{2}\left(t, v_{2}\right)$ can be obtained, respectively, as

$$
\begin{aligned}
& \boldsymbol{n}_{1}\left(t, v_{1}\right)=\boldsymbol{a}_{0}(t) \times\left(k^{\prime}(t)+v_{1} \boldsymbol{a}_{0}^{\prime}(t)\right), \\
& \boldsymbol{n}_{2}\left(t, v_{2}\right)=\boldsymbol{a}_{2}(t) \times\left(k^{\prime}(t)+v_{2} \boldsymbol{a}_{2}^{\prime}(t)\right) .
\end{aligned}
$$

Since the surfaces $\Phi_{1}\left(t, v_{1}\right)$ and $\Phi_{2}\left(t, v_{2}\right)$ intersect along the common base curve $k(t)$ if $v_{1}=v_{2}=0$, we get the normal vectors $\boldsymbol{n}_{1}\left(t, v_{1}\right)$ and $\boldsymbol{n}_{2}\left(t, v_{2}\right)$ along the base curve $k(t)$ as

$$
\begin{aligned}
& \boldsymbol{n}_{1}(t, 0)=\boldsymbol{a}_{0}(t) \times k^{\prime}(t), \\
& \boldsymbol{n}_{2}(t, 0)=\boldsymbol{a}_{2}(t) \times k^{\prime}(t),
\end{aligned}
$$

for all $t \in I$. Then, we obtain the scalar product of these vectors as

$$
\left\langle\boldsymbol{n}_{1}(t, 0), \boldsymbol{n}_{2}(t, 0)\right\rangle=-\left\langle\boldsymbol{a}_{0}(t), k^{\prime}(t)\right\rangle\left\langle k^{\prime}(t), \boldsymbol{a}_{2}(t)\right\rangle .
$$

This means that $\boldsymbol{n}_{1}$ and $\boldsymbol{n}_{2}$ are perpendicular along $k(t)$ if and only if $\left\langle\boldsymbol{a}_{0}(t), k^{\prime}(t)\right\rangle=0$ or $\left\langle k^{\prime}(t), \boldsymbol{a}_{2}(t)\right\rangle=0$.

Proposition 3.6. Consider two ruled surfaces $\Phi_{1}\left(t, v_{1}\right)=k(t)+v_{1} \boldsymbol{a}_{0}(t)$ and $\Phi_{2}\left(t, v_{2}\right)=$ $k(t)+v_{2} \boldsymbol{a}_{2}(t)$ corresponding to the hyper-dual curve $\tilde{\Gamma}(t) \in \mathbb{S}_{\tilde{\mathbb{D}}_{1}}^{2}$ such that their normal vectors are perpendicular along their common base curve $k(t)$. If $k(t)$ is the principal curve of $\Phi_{1}\left(t, v_{1}\right)$ (resp., $\Phi_{2}\left(t, v_{2}\right)$ ), then $k(t)$ is also the principal curve of $\Phi_{2}\left(t, v_{2}\right)$ (resp., $\left.\Phi_{1}\left(t, v_{2}\right)\right)$. 
Proof. Let $k(t)$ be a curve both on the surfaces $\Phi_{1}\left(t, v_{1}\right)=k(t)+v_{1} \boldsymbol{a}_{0}(t)$ and $\Phi_{2}\left(t, v_{2}\right)=$ $k(t)+v_{2} \boldsymbol{a}_{2}(t)$. And assume that the Darboux frames (see [15]) along the curve $k(t)$ on $\Phi_{1}\left(t, v_{1}\right)$ and $\Phi_{2}\left(t, v_{2}\right)$ are, respectively, $\left\{\boldsymbol{t}_{1}(t), \boldsymbol{y}_{1}(t), \boldsymbol{n}_{1}(t)\right\}$ and $\left\{\boldsymbol{t}_{2}(t), \boldsymbol{y}_{2}(t), \boldsymbol{n}_{2}(t)\right\}$, that means

$$
\begin{aligned}
\boldsymbol{t}_{1}(t, 0) & =\boldsymbol{t}_{2}(t)=\frac{d}{d t} k(t)=k^{\prime}(t)=\boldsymbol{t}(t), \\
\boldsymbol{n}_{1}(t, 0) & =\boldsymbol{a}_{0}(t) \times k^{\prime}(t)=\boldsymbol{a}_{0}(t) \times \boldsymbol{t}(t), \\
\boldsymbol{n}_{2}(t, 0) & =\boldsymbol{a}_{2}(t) \times k^{\prime}(t)=\boldsymbol{a}_{2}(t) \times \boldsymbol{t}(t), \\
\boldsymbol{y}_{1}(t, 0) & =\boldsymbol{n}_{1}(t, 0) \times \boldsymbol{t}_{1}(t)=\boldsymbol{n}_{1}(t, 0) \times \boldsymbol{t}(t), \\
\boldsymbol{y}_{2}(t, 0) & =\boldsymbol{n}_{2}(t, 0) \times \boldsymbol{t}_{2}(t)=\boldsymbol{n}_{2}(t, 0) \times \boldsymbol{t}(t) .
\end{aligned}
$$

Moreover, we have

$$
\begin{aligned}
\frac{d}{d t} \boldsymbol{n}_{1}(t, 0) & =-k_{n_{1}} \boldsymbol{t}(t)-t_{g_{1}} \boldsymbol{y}_{1}(t, 0), \\
\frac{d}{d t} \boldsymbol{n}_{2}(t, 0) & =-k_{n_{2}} \boldsymbol{t}(t)-t_{g_{2}} \boldsymbol{y}_{2}(t, 0),
\end{aligned}
$$

where $k_{n_{1}}, k_{n_{2}}$ are the normal curvatures and $t_{g_{1}}, t_{g_{2}}$ are the geodesic torsions. If $t_{g_{1}}=0$ or $t_{g_{2}}=0$, then $k(t)$ is a principal curve. Since the normal vectors $\boldsymbol{n}_{1}$ and $\boldsymbol{n}_{2}$ are perpendicular,

$$
\left\langle\boldsymbol{n}_{1}(t, 0), \boldsymbol{n}_{2}(t, 0)\right\rangle=0 .
$$

By taking the derivative of this equation, we get

$$
\frac{d}{d t}\left\langle\boldsymbol{n}_{1}(t, 0), \boldsymbol{n}_{2}(t, 0)\right\rangle=\left\langle\frac{d}{d t} \boldsymbol{n}_{1}(t, 0), \boldsymbol{n}_{2}(t, 0)\right\rangle+\left\langle\boldsymbol{n}_{1}(t, 0), \frac{d}{d t} \boldsymbol{n}_{2}(t, 0)\right\rangle .
$$

Using Eqs. (3.40-42), we obtain

$$
\left\langle-k_{n_{1}} \boldsymbol{t}(t)-t_{g_{1}} \boldsymbol{y}_{1}(t, 0), \boldsymbol{n}_{2}(t, 0)\right\rangle+\left\langle\boldsymbol{n}_{1}(t, 0),-k_{n_{2}} \boldsymbol{t}(t)-t_{g_{2}} \boldsymbol{y}_{2}(t, 0)\right\rangle=0 .
$$

And since $\left\langle\boldsymbol{n}_{1}(t, 0), \boldsymbol{t}(t)\right\rangle=\left\langle\boldsymbol{t}(t), \boldsymbol{n}_{2}(t, 0)\right\rangle=0$, we get

$$
-t_{g_{1}}\left\langle\boldsymbol{y}_{1}(t, 0), \boldsymbol{n}_{2}(t, 0)\right\rangle-t_{g_{2}}\left\langle\boldsymbol{n}_{1}(t, 0), \boldsymbol{y}_{2}(t, 0)\right\rangle=0 .
$$

That is

$$
-t_{g_{1}}\left\langle\boldsymbol{y}_{1}(t), \boldsymbol{n}_{2}(t)\right\rangle=t_{g_{2}}\left\langle\boldsymbol{n}_{1}(t), \boldsymbol{y}_{2}(t)\right\rangle .
$$

As a result, if $t_{g_{1}}=0$ (resp. $\left.t_{g_{2}}=0\right)$, then $t_{g_{2}}=0$ (resp. $\left.t_{g_{1}}=0\right)$. And this completes the proof.

\section{Examples of ruled surfaces constructed by curves on $\mathbb{S}_{\tilde{\mathbb{D}}_{1}}^{2}$}

Example 4.1. Let us take the hyper-dual curve $\tilde{\Gamma}(t)=\hat{A}(t)+\varepsilon^{*} \hat{A}^{*}(t)$, where $\hat{A}(t)=$ $\boldsymbol{a}_{0}(t)+\varepsilon \boldsymbol{a}_{1}(t)$ and $\hat{A}^{*}(t)=\boldsymbol{a}_{2}(t)+\varepsilon \boldsymbol{a}_{3}(t)$. Here;

$$
\begin{aligned}
& \boldsymbol{a}_{0}(t)=(\cos t \cos 2 t, \cos t \sin 2 t, \sin t), \\
& \boldsymbol{a}_{1}(t)=(\sin t \sin 2 t,-\sin t \cos 2 t, 0), \\
& \boldsymbol{a}_{2}(t)=(\sin t \cos 2 t, \sin t \sin 2 t,-\cos t), \\
& \boldsymbol{a}_{3}(t)=(-\cos t \sin 2 t, \cos t \cos 2 t, 0) .
\end{aligned}
$$

Since $|\hat{A}(t)|_{D}=\left|\hat{A}^{*}(t)\right|_{D}=1$ and $\left\langle\hat{A}(t), \hat{A}^{*}(t)\right\rangle_{D}=0 ; \tilde{\Gamma}(t)$ is a hyper-dual curve on $\mathbb{S}_{\tilde{\mathbb{D}}_{1}}^{2}$, and $\hat{A}(t)$ and $\hat{A}^{*}(t)$ are dual curves on unit dual sphere $\mathbb{S}_{\mathbb{D}}^{2}$. Using Eqs. (3.23) and 
(3.24), the ruled surfaces corresponding to the dual curves $\hat{A}(t)=\boldsymbol{a}_{0}(t)+\varepsilon \boldsymbol{a}_{1}(t)$ and $\hat{A}^{*}(t)=\boldsymbol{a}_{2}(t)+\varepsilon \boldsymbol{a}_{3}(t)$ are obtained, respectively, as

$$
\begin{aligned}
\Phi_{1}\left(t, u_{1}\right) & =\left(\sin ^{2} t \cos 2 t, \sin ^{2} t \sin 2 t,-\sin t \cos t\right) \\
& +u_{1}(\cos t \cos 2 t, \cos t \sin 2 t, \sin t) \\
\Phi_{2}\left(t, u_{2}\right) & =\left(\cos ^{2} t \cos 2 t, \cos ^{2} t \sin 2 t, \sin t \cos t\right) \\
& +u_{2}(\sin t \cos 2 t, \sin t \sin 2 t,-\cos t),
\end{aligned}
$$

where $t \in I=(0, \pi)$ and $u_{1}, u_{2} \in \mathbb{R}$. For $t=t_{0}, \Phi_{1}\left(t_{0}, u\right)$ and $\Phi_{2}\left(t_{0}, u\right)$ represent the lines $m_{t_{0}}\left(u_{1}\right)$ and $n_{t_{0}}\left(u_{2}\right)$, respectively. Moreover, $m_{t_{0}}\left(u_{1}\right)$ is a line corresponding to the unit dual vector $\hat{A}\left(t_{0}\right)=\boldsymbol{a}_{0}\left(t_{0}\right)+\varepsilon \boldsymbol{a}_{1}\left(t_{0}\right)$, and $n_{t_{0}}\left(u_{2}\right)$ is a line corresponding to the unit dual vector $\hat{A}^{*}\left(t_{0}\right)=\boldsymbol{a}_{2}\left(t_{0}\right)+\varepsilon \boldsymbol{a}_{3}\left(t_{0}\right)$.

For all $t \in I$, the intersection point of the lines $m_{t}\left(u_{1}\right)$ and $n_{t}\left(u_{2}\right)$ will be obtained as

$$
k(t)=(\cos 2 t, \sin 2 t, 0),
$$

where $u_{1}=\cos t$ and $u_{2}=\sin t$. Using Eqs. (3.28) and (3.29), these ruled surfaces can be expressed as

$$
\begin{aligned}
& \Phi_{1}\left(t, v_{1}\right)=(\cos 2 t, \sin 2 t, 0)+v_{1}(\cos t \cos 2 t, \cos t \sin 2 t, \sin t), \\
& \Phi_{2}\left(t, v_{2}\right)=(\cos 2 t, \sin 2 t, 0)+v_{2}(\sin t \cos 2 t, \sin t \sin 2 t,-\cos t),
\end{aligned}
$$

where $v_{1}, v_{2} \in \mathbb{R}$. From Eqs. (4.8) and (4.9), it can be seen that the ruled surfaces $\Phi_{1}\left(t, v_{1}\right)$ and $\Phi_{2}\left(t, v_{2}\right)$ have a common base curve $k(t)=(\cos 2 t, \sin 2 t, 0)$. Using Eqs. (4.1) and (4.3), we get $\left\langle\boldsymbol{a}_{0}(t), \boldsymbol{a}_{2}(t)\right\rangle=0$. Thus, the position vectors of the director curves $\boldsymbol{a}_{0}(t)$ and $\boldsymbol{a}_{2}(t)$ of the surfaces $\Phi_{1}\left(t, v_{1}\right)$ and $\Phi_{2}\left(t, v_{2}\right)$ are perpendicular.

The velocity vector $k^{\prime}(t)=(-2 \sin 2 t, 2 \cos 2 t, 0)$ is perpendicular to $\boldsymbol{a}_{0}(t)$ and $\boldsymbol{a}_{2}(t)$. Thus, according to Theorem 3.5 the normal vectors of $\Phi_{1}\left(t, v_{1}\right)$ and $\Phi_{2}\left(t, v_{2}\right)$ are perpendicular along $k(t)$.

$\Phi_{1}\left(t, v_{1}\right)$ and $\Phi_{2}\left(t, v_{2}\right)$ represent Möbius strips. For intervals $0 \leq t \leq \pi,-0.3 \leq v_{1} \leq 0.3$ and $-0.3 \leq v_{2} \leq 0.3$, these two Möbius strips can be drawn as in Fig. 3 .

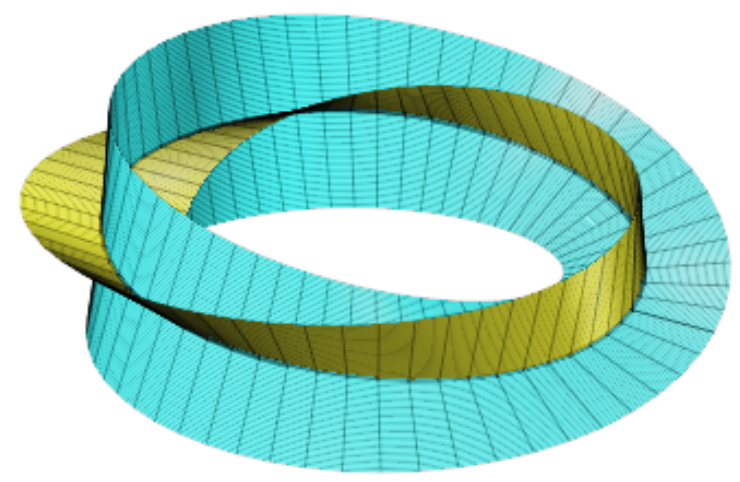

Figure 3. Geometric representation of two Möbius strips in $\mathbb{R}^{3}$ corresponding to the hyper-dual curve $\tilde{\Gamma}(t)$ on $\mathbb{S}_{\tilde{\mathbb{D}}_{1}}^{2}$. 
Example 4.2. Let us take the hyper-dual curve $\tilde{\Gamma}(t)=\hat{A}(t)+\varepsilon^{*} \hat{A}^{*}(t)$, where $\hat{A}(t)=$ $\boldsymbol{a}_{0}(t)+\varepsilon \boldsymbol{a}_{1}(t)$ and $\hat{A}^{*}(t)=\boldsymbol{a}_{2}(t)+\varepsilon \boldsymbol{a}_{3}(t)$. Here;

$$
\begin{aligned}
& \boldsymbol{a}_{0}(t)=(0,0,1), \\
& \boldsymbol{a}_{1}(t)=(\sin t,-\cos t, 0), \\
& \boldsymbol{a}_{2}(t)=(\cos t, \sin t, 0), \\
& \boldsymbol{a}_{3}(t)=(-t \sin t, t \cos t, 0) .
\end{aligned}
$$

Since $|\hat{A}(t)|_{D}=\left|\hat{A}^{*}(t)\right|_{D}=1$ and $\left\langle\hat{A}(t), \hat{A}^{*}(t)\right\rangle_{D}=0 ; \tilde{\Gamma}(t)$ is a curve on $\mathbb{S}_{\tilde{\mathbb{D}}_{1}}^{2}$, and $\hat{A}(t)$ and $\hat{A}^{*}(t)$ are dual curves on unit dual sphere $\mathbb{S}_{\mathbb{D}}^{2}$. Using Eqs. (3.23) and (3.24), the ruled surfaces corresponding to the dual curves $\hat{A}(t)=\boldsymbol{a}_{0}(t)+\varepsilon \boldsymbol{a}_{1}(t)$ and $\hat{A}^{*}(t)=\boldsymbol{a}_{2}(t)+\varepsilon \boldsymbol{a}_{3}(t)$ are obtained, respectively, as

$$
\begin{aligned}
& \Phi_{1}\left(t, u_{1}\right)=(\cos t, \sin t, 0)+u_{1}(0,0,1), \\
& \Phi_{2}\left(t, u_{2}\right)=(0,0, t)+u_{2}(\cos t, \sin t, 0),
\end{aligned}
$$

where $t \in I=(0, \pi)$ and $u_{1}, u_{2} \in \mathbb{R}$. From the Theorem 3.4, the ruled surfaces $\Phi_{1}\left(t, u_{1}\right)$ and $\Phi_{2}\left(t, u_{2}\right)$ can be also obtained, respectively, as

$$
\begin{array}{ll}
\Phi_{1}\left(t, v_{1}\right)=(\cos t, \sin t, t)+v_{1}(0,0,1), & v_{1} \in \mathbb{R} \\
\Phi_{2}\left(t, v_{2}\right)=(\cos t, \sin t, t)+v_{2}(\cos t, \sin t, 0), & v_{2} \in \mathbb{R}
\end{array}
$$

where $k(t)=(\cos t, \sin t, t)$ is a common base curve of $\Phi_{1}\left(t, v_{1}\right)$ and $\Phi_{2}\left(t, v_{2}\right)$. Since $\left\langle\boldsymbol{a}_{0}(t), \boldsymbol{a}_{2}(t)\right\rangle=0$, the position vectors of the director curves $\boldsymbol{a}_{0}(t)=(0,0,1)$ and $\boldsymbol{a}_{2}(t)=$ $(\cos t, \sin t, 0)$ are perpendicular.

The velocity vector $k^{\prime}(t)=(-\sin t, \cos t, 1)$ is perpendicular to $\boldsymbol{a}_{2}(t)$. Thus, according to Theorem 3.5 the normal vectors of $\Phi_{1}\left(t, v_{1}\right)$ and $\Phi_{2}\left(t, v_{2}\right)$ are perpendicular along $k(t)$.

$\Phi_{1}\left(t, v_{1}\right)$ and $\Phi_{2}\left(t, v_{2}\right)$ represent, respectively, cylindrical and helicoid surfaces. They intersect along a helix curve $k(t)=(\cos t, \sin t, t)$. For intervals $-\pi \leq t \leq \pi,-10 \leq v_{1} \leq 10$ and $-10 \leq v_{2} \leq 10$, these surfaces can be drawn as in Fig. 4 .

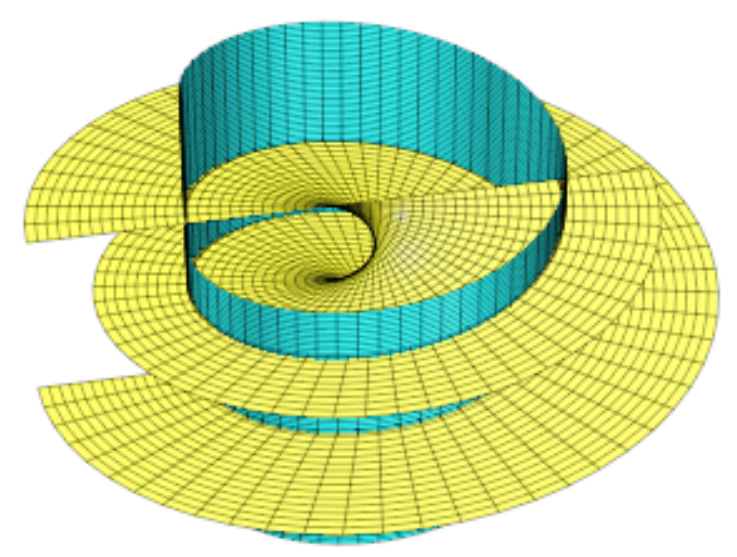

Figure 4. Geometric representation of two ruled surfaces in $\mathbb{R}^{3}$ corresponding to the hyper-dual curve $\tilde{\Gamma}(t)$ on $\mathbb{S}_{\tilde{\mathbb{D}}_{1}}^{2}$.

\section{Conclusions}

In this paper, some basic concepts of hyper-dual numbers are given by using dual numbers. Using these concepts, we have given the definition of a set $\mathbb{S}_{\tilde{\mathbb{D}}_{1}}^{2}$, which is a subset of unit hyper-dual sphere $\mathbb{S}_{\tilde{\mathbb{D}}}^{2}$. We show that there exists a one-to-one correspondence between the points of $\mathbb{S}_{\tilde{\mathbb{D}}_{1}}^{2}$ and any two intersecting perpendicular directed lines in $\mathbb{R}^{3}$. 
Moreover, we show that each hyper-dual curve on $\mathbb{S}_{\tilde{\mathbb{D}}_{1}}^{2}$ represents two ruled surfaces in $\mathbb{R}^{3}$ such that these ruled surfaces intersect along a common base curve.

\section{References}

[1] O.P. Agrawal, Hamilton operators and dual-number-quaternions in spatial kinematics, Mech. Mach. Theory, 22 (6), 569-575, 1987.

[2] W.K. Clifford, Preliminary sketch of biquaternions, Proc. London Math. Soc. 4 (64), 381-395, 1873.

[3] A. Cohen and M. Shoham, Application of hyper-dual numbers to multi-body kinematics, J. Mech. Robot., 8 (1), 011015, (4 pages), 2016.

[4] A. Cohen and M. Shoham, Application of hyper-dual numbers to rigid bodies equations of motion, Mech. Mach. Theory 111, 76-84, 2017.

[5] A. Cohen and M. Shoham, Principle of transference-An extension to hyper-dual numbers, Mech. Mach. Theory 125, 101-110, 2018.

[6] J.A. Fike, Numerically exact derivative calculations using hyper-dual numbers, 3rd Annual Student Joint Workshop in Simulation-Based Engineering and Design, 2009.

[7] J.A. Fike and J.J. Alonso, The development of hyper-dual numbers for exact secondderivative calculations, 49th AIAA Aerodpace Sciences Meeting including the New Horizons Forum and Aerospace Exposition, 4-7, 2011.

[8] J.A. Fike and J.J. Alonso, Automatic differentiation through the use of hyper-dual numbers for second derivatives, in: Lecture Notes in Computational Science and Engineering, 87 (201), 163-173, 2011.

[9] J.A. Fike, S. Jongsma, J.J. Alonso, and E. van der Weida, Optimization with gradient and hessian information calculated using hyper-dual numbers, 29 AIAA Applied Aerodynamics Conference, 2011.

[10] F. Hathout, M. Bekar, and Y. Yayl, Ruled surfaces and tangent bundle of unit 2sphere, Int. J. Geom. Methods Mod. Phys., 14 (10), 1750145, 2017.

[11] A.P. Kotelnikov, Screw calculus and some applications to geometry and mechanics, Annal. Imp. Univ. Kazan, Russia, 1895.

[12] J.M. McCarthy, The Instantaneous Kinematics of Line Trajectories in Terms of a Kinematic Mapping of Spatial Rigid Motion, ASME J. Mech., Transm., Autom. Des. 109 (1), 95-100, 1987.

[13] J.M. McCarthy, On the Scalar and Dual Formulations of the Curvature Theory of Line Trajectories, ASME J. Mech., Transm., Autom. Des. 109, 101-106, 1987.

[14] Y.S. Oh, P. Abhishesh, and B.S. Ryuh, Study on Robot Trajectory Planning by Robot EndEffector Using Dual Curvature Theory of the Ruled Surface, World Academy of Science, Engineering and Technology, International Journal of Mechanical, Aerospace, Industrial, Mechatronic and Manufacturing Engineering 11 (3), 577-582, 2017.

[15] B. O'Neill, Elementary Differential Geometry, Revised 2nd edition, Academic Press, USA, 2006.

[16] M. Önder and H.H. Uğurlu, Normal and Spherical Curves in Dual Space $\mathbb{D}^{3}$, Mediterr. J. Math. 10, 1527-1537, 2013.

[17] B.S. Ryuh and G.R. Pennock, Accurate motion of a robot end-effector using the curvature theory of ruled surfaces, Journal of Mechanisms, Transmissions and Automation in Design 110 (4), 383-388, 1988.

[18] K. Sprott and B. Ravani, Kinematic generation of ruled surfaces, Adv. Comput. Math. 17, 115-133, 2002.

[19] E. Study, Geometry der Dynamen, Leipzig, 1901.

[20] G.R. Veldkamp, On the use of dual numbers, vectors and matrices in instantaneous, spatial kinematics, Mech. Mach. Theory 11 (2), 141-156, 1976. 